A. Pramesh Rao, G. Swarup and Gopal-Krishna, eds.

\title{
Study of Objects of Low Radio Frequency Catalogues and IRAS Data - Cross-Identification
}

\author{
O.V.Verkhodanov and S.A.Trushkin \\ Special Astrophysical Observatory, Nizhnij Arkhyz, Russia, 369167
}

\begin{abstract}
We present results of cross-identifications of the objects from two large catalogues, IRAS and the low radio frequency Texas catalogue at $365 \mathrm{MHz}$ in the database CATS. The resulting list consists of 715 pairs of sources within a circle of radius of 60 arcsec. It has been cross-identified with the GB6 $4.85 \mathrm{GHz}$, NVSS $1.4 \mathrm{GHz}$, FIRST $1.4 \mathrm{GHz}$, WENSS $325 \mathrm{MHz}$ radio catalogues, ROSAT catalogues and different optical data lists, including APM catalogue. We selected 3 subsamples: objects matching in $3^{\prime \prime}$ radius, ultra steep spectrum (USS) radio sources, inverse spectrum radio sources, galactic sources. Several objects have radio spectral index $\alpha<-2$, some of them in Galactic plane.
\end{abstract}

\section{Introduction}

Exploration of cosmic objects in different frequency ranges is the only way of understanding their physical nature. To find additional data, astronomers usually use the large astrophysical catalogues containing results of different kinds of observations. The most interesting things can be found in cross-identifying objects from different catalogues. The database CATS (http://cats.sao.ru), including more than 300 astrophysical catalogues, has been organized and relevant software has been worked out (Verkhodanov et al., 1997). Thus some functions of this database could be used in search and selection of objects. We have used two catalogues, the IRAS (Point, Faint and Serendipitous) Source catalogue (Beichman et al., 1988; IRAS, 1985) and the Texas $365 \mathrm{MHz}$ catalogue (Douglas et al., 1996), to carry out cross-identification and to form an initial source identification list for subsequent investigation.

A sample of objects from the IRAS Faint Sources Catalogue has been partly radio-mapped and studied by Condon and Broderick (1991). Below we use their infrared criteria of selection of galaxies.

One of the aims of the work is an attempt to detect sources with unusual spectra or ratio of infrared and radio fluxes. In the future we propose to study them with the RATAN-600 radio telescope. Part of these sources in the Galactic plane could be identified with sources from the new Galactic survey, carried out by Trushkin (1995). 


\section{Cross-identification}

The procedure of cross-identification consisted in matching the IRAS sources to the Texas sources by seaching for their coincident positions. The position uncertainties of each object have not been taken into account because they vary from 40 to $80^{\prime \prime}$ for the IRAS coordinates. The Texas sources have a high coordinate accuracy, about $1^{\prime \prime}$, but position shifts of interferometric sidelobes up to $52^{\prime \prime}$ are possible in these measurements (Douglas et al., 1996). Here it is more preferable to retain any small portion of false identifications than the lost true ones. Thus we have concluded that the search radius of $60^{\prime \prime}$ is acceptable for initial statistical consideration. Probably a portion of cross-identifications is occasional. The resulting list we have identified through all the catalogues of CATS database to estimate spectral index distribution and to find existing counterparts.

\section{Some Results}

As a result after the cross-identification we have obtained a list of 715 sources of the IRAS and Texas catalogues (ITA catalogue: IRAS-Texas associations). One Texas source is associated with two different IRAS sources in the extended $\left(6^{\prime} \times 8^{\prime}\right)$ Crab nebula.

Flux density distributions showed that most ITA sources have radio flux densities less than $5 \mathrm{Jy}$, while IR fluxes vary from tens of Jy to several thousand Jy. It has been found that the strong IR sources in the Galactic plane with the flux ratio $R=S_{I R A S} / S_{\text {radio }}>500$, where $S_{I R A S}$ is the flux at $60-100 \mu m$ and $S_{\text {radio }}$ is the flux at centimeter or decimeter wavelengths, are associated with the galactic HII regions, while non-thermal galactic sources, known as supernova remnants, have $R<100$. (Broadbent et al., 1989). The ITA catalogue has 45 sources with $R>500$. All of them have been identified with HII regions, which confirms the previous conclusion. For the ITA sources with $S_{60}>100 \mathrm{Jy}$ we have examined the behaviour of $S_{365}$ vs $S_{60}$ as shown in Fig.1a. Considerable correlation coefficient $(z 0.6)$ is presented only for the IRAS sources with $S_{60}>$ $500 \mathrm{Jy}$. Usually the strong IR radiation is emitted by dust embedded in HII regions and the correlation can be due to the same source of energy. The radio continuum emission and infrared emission are proportional to the recombination rate, and for the electron temperature $T_{e}=7000 \mathrm{~K}$ the ratio of emissivities at $60 \mu m$ and at $2.7 \mathrm{GHz}$ is $\epsilon(60 \mu m / \epsilon(2.7 G H z)=190 f$, where $f$ is the infrared excess, the ratio of the total IR luminosity to $L_{\alpha}$ luminosity has been found to be equal to 3-4 for typical HII regions (Broadbent et al., 1989). For optically thin HII region this ratio is almost the same, but for optically thick HII regions this ratio is still higher at $365 \mathrm{MHz}$.

After the cross-identification of the ITA catalogue with the CATS data (WENSS $325 \mathrm{MHz}$, MIYUN $232 \mathrm{MHz}$, Cambridge catalogues, NVSS $1.4 \mathrm{GHz}$, FIRST $1.4 \mathrm{GHz}, \mathrm{GB} 64.85 \mathrm{GHz}$, etc) we have obtained continual spectra by several points almost for all ITA objects and constructed distributions of a number of sources vs spectral index $\alpha\left(\mathrm{S} \propto \nu^{\alpha}\right)$ for the 260 identified sources (Fig.1b).

Distributions of spectral indices for sources with low and high galactic latitudes showed a portion of steep spectrum sources $(\alpha<-0.8)$ is smaller in latter 

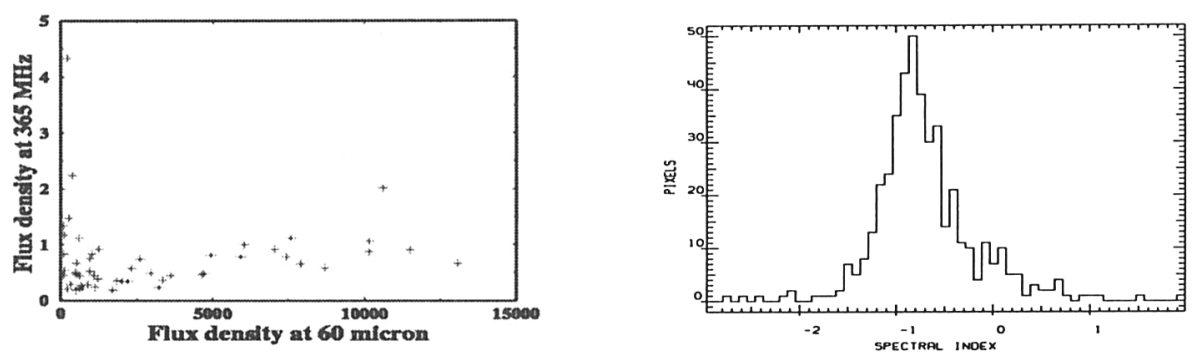

Figure 1. Behaviour of $S_{365}$ vs $S_{60}$ for ITA sources with $S_{60}>100 \mathrm{Jy}$ (left panel). Distribution of spectral index for PSC subsamples at $5 \mathrm{GHz}$ (right panel)

case in the second figure than in the former, while the relative number of flat spectrum sources $(\alpha>-0.5)$ has increased for the sample of the galactic plane sources. Obviously the thermal galactic radio sources have changed statistics in the low galactic latitude region. Some sources with inverse spectra seem to be optically thick HII regions.

The 97 ITA sources fall in the region $|l|<17^{\circ}$ and $|b|<5.5^{\circ}$ of the Galactic survey by Trushkin (1995). All the 63 ITA sources with $S_{365}>0.4 \mathrm{Jy}$ have been found at $3900 \mathrm{MHz}$. Normally these sources are compact with sizes $<2^{\prime}$, but 15 sources seem to be associated with extended ones. Ten bright PSC sources with $R>500$ are bright radio sources at $3900 \mathrm{MHz}$, which have at this frequency a flux density much higher than at $365 \mathrm{MHz}$. Thus, they seem to be optically thick HII regions.

For the identification of infrared sources with galaxies we have used the following criteria: $[\alpha(25 \mu m, 60 \mu m) z+1.5]$ and $|b|>10$ (Condon and Broderick, 1991). 138 of ITA sources satisfy these criteria. This sample has $R<200$ and the mean value of fluxes are equal to: $S_{365}=0.6 \pm 0.6, S_{60}=31 \mathrm{Jy}$. 91 ITA sources have already been identified with galaxies in the IRAS point source catalogue.

Cross-identfication with miscellaneous optical and mixed catalogues (PGC, MCG, AGN, Dixon, etc.) permitted us to detect 347 identifications including 14 Markarian galaxies and 87 Zwicky galaxies. 42 objects have been found in the Veron-Veron catalogue of active nuclei galaxies and QSOs. 110 sources have counterparts in the X-ray ROSAT catalogues, where only 4 are located in the region of $|b|<10^{\circ}$. Others are connected with AGN, having IR and low frequency radio emission.

Using ITA catalogue of 715 objects we selected 3 main subsamples which can be unteresting for further study:

- USS $\alpha<-1$ : 128 objects (37 in the Galactic plane), 6 with $\alpha<-2$

- Inverse Spectrum sources: 27 objects (18 in the Galactic plane), being HII regions. 
- and sources with radio-infrared coordinates coincidence within circle of $3^{\prime \prime}$ radius.

At the first step we started from the $3^{\prime \prime}$ coincident sources. In Table 1 we show statistics of distribution of spectrum type.

Table 1. Sources spectra statistics, identified within $3^{\prime \prime}$

\begin{tabular}{|lllcr|}
\hline Spectrum type & & curve type & number & $\%$ \\
\hline \hline Inverse $(0<\alpha)$ & IS & $+A+B x$ & 8 & 3.5 \\
\hline Flat $(-0.8<\alpha<0)$ & FS & $+A-B x$ & 58 & 25.3 \\
\hline Steep $(\alpha<-0.8)$ & SS & $+A-B x$ & 51 & 22.3 \\
ultra steep $(\alpha<-1.0)$ & USS & & 18 & 7.9 \\
\hline Concave & $C^{-}{ }_{1} \pm A-B x+C x^{2}$ & 34 & 14.8 \\
& $C^{-}{ }^{2}$ & $\pm A \pm B x+C e^{-x}$ & 5 & 2.2 \\
& $C^{-}{ }_{3}$ & $\pm A \pm B x+C e^{x}$ & 1 & 0.4 \\
\hline Convex & $C^{+}{ }_{1} \pm A \pm B x-C x^{2}$ & 57 & 24.8 \\
gigahertz peaked & $\mathrm{GPS}$ & & 4 & 1.8 \\
& $C^{+}{ }^{2}$ & $\pm A \pm B x-C e^{-x}$ & 2 & 0.9 \\
& $C^{+}{ }_{3}$ & $\pm A \pm B x-C e^{x}$ & 2 & 0.9 \\
\hline Complex spectrum & $\mathrm{E}$ & & 2 & 0.9 \\
\hline only 1 point & & & 9 & 3.9 \\
\hline
\end{tabular}

A program of USS ITA source observation started at RATAN-600. One of these objects, IRAS F02044+0957, having radio spectral index $\alpha=-1.2$ was observed with INAOE 2.1-m telescope (Mexico) by V.Chavushian. It is a pair of interacrive emission line galaxies at $z=0.1$.

New data obtained from RATAN-600 and INAOE observations and from PSCz catalogue (Saunders et al., 1999) will help us to make detailed classification an study of ITA sources.

Authors are thankful to Vahram Chavushyam for useful discussion.

\section{References}

Beichman C.A. et al. 1988, NASA RP-1190, Infrared Astronomical Satellite (IRAS), Catalogs and Atlases, 1, Explanatory Supplement.

Broadbent A., Haslam C.G.T., Osborne J.L.: 1989, MNRAS, 237, 381.

Condon J.J., Broderick J.J.: 1991, AJ, 102, 1663.

Douglas J.N. et al. 1996, AJ, 111, 1945.

IRAS. 1985, IRAS point sources catalog, Joint IRAS Science Working Group.

Saunders W. et al. 1999. astro-ph/0001117

Trushkin S.A.: 1995, Astron. Asrtophys. Trans. (in press).

Verkhodanov O.V. et al. 1997 in ASP Conf. Ser. Vol. 125, "Astronomical Data Analysis Software and Systems VI", ed. G.Hunt \& H.E.Payne. ASP Conference Series, 322. http://cats.sao.ru 